\title{
Tsafon
}

Revue d'études juives du Nord

$76 \mid 2018$

Expressions yiddish de la nature

\section{La nature, point d'ancrage et de gravité de l'œuvre littéraire de Moyshe Kulbak (1896-1937)}

Arnaud Bikard

\section{(2) OpenEdition}

1 Journals

Édition électronique

URL : https://journals.openedition.org/tsafon/1227

DOI : $10.4000 /$ tsafon. 1227

ISSN : 2609-6420

Éditeur

Association Jean-Marie Delmaire

Édition imprimée

Date de publication : 1 décembre 2018

Pagination : 51-68

ISSN : 1149-6630

\section{Référence électronique}

Arnaud Bikard, « La nature, point d'ancrage et de gravité de l'œuvre littéraire de Moyshe Kulbak

(1896-1937) », Tsafon [En ligne], 76 | 2018, mis en ligne le 30 juin 2019, consulté le 25 juin 2021. URL http://journals.openedition.org/tsafon/1227 ; DOl : https://doi.org/10.4000/tsafon.1227

Tsafon. Revues d'études juives du Nord 


\title{
La nature, point d'ancrage et de gravité de l'œuvre littéraire de Moyshe Kulbak (1896-1937)
}

\begin{abstract}
Arnaud Bikard*
Moyshe Kulbak, écrivain yiddish né à Smarhon, dans l'actuelle Biélorussie, est connu aujourd'hui principalement pour ses créations en prose : ses deux récits modernistes, Moshiekh Ben Efrayim [Le Messie, fils d'Ephraïm] et Montog [Lundi], composés dans l'effervescence créative des années 1920 ou sa grande fresque familiale Di Zelminyaner [Les Zelminiens], datant essentiellement du début des années 1930, qui dépeint avec un humour dévastateur l'entrée dans la modernité soviétique d'une famille juive de Minsk ${ }^{1}$. Sa vie adulte, passée dans les grands centres urbains d'Europe orientale, essentiellement Vilnius et Minsk, auxquels s'ajoute la parenthèse cruciale d'un séjour à Berlin entre 1920 et 1923, explique l'écho perceptible dans son œuvre des grands bouleversements esthétiques de son temps - expressionnisme, futurisme puis réalisme soviétique.

Or, Kulbak, pour ses contemporains, était avant tout un poète, largement reconnu et admiré pour ses vers, source première de sa création. Et dans sa poésie, toute moderne qu'elle soit dans sa forme et dans sa vision, Kulbak est essentiellement un écrivain de la nature, dépeignant sa région natale avec une originalité soulignée à maintes

\footnotetext{
${ }^{*}$ Inalco, Paris.

Cette recherche a été soutenue par la Fondation Russe des Sciences, projet 15-1800062, « Formation of culture in diaspora », Université d’État de Saint-Pétersbourg.

${ }^{1}$ Moyshe Kulbak, Le Messie, fils d'Éphraïm, trad. Carole Ksiazenicer-Matheron, Imprimerie nationale, 1995; Id., Lundi, trad. Bernard Vaisbrot, Lausanne, L'Âge d'Homme, 1982 ; Id., Les Zelminiens, trad. Régine Robin, Paris, Seuil, 1988.
} 
reprises, en particulier par la critique de langue yiddish. C'est ainsi que Zalmen Reyzen salue en lui le poète « de la 'terrestrialité' [erdishkayt] primitive, saine et fraîche, qui dévoile à nos yeux une nouvelle judéité colorée sur le fond gris du paysage lituanien-biélorusse - des figures classiques de simples Juifs-de-village, Juifs-de-la-nature, dont émane l'odeur brute et épicée du champ, de la forêt, de la rivière $»^{2}$. L'emphase avec laquelle cette caractéristique de son écriture est ici soulignée, la création de concepts ad-hoc pour désigner les juifs ainsi représentés, montre à quel point le critique voit dans cette qualité poétique une nouveauté bienvenue ${ }^{3}$. Littéralement adulé par la jeunesse de Vilnius, où il a enseigné la littérature de 1924 à 1928, Kulbak a laissé une marque essentielle sur toute une génération et la place cruciale que joue la nature chez les membres du mouvement Yung Vilne [jeune Vilnius] est, de l'avis même de l'un d'entre eux, due à l'influence de celui qu'ils considéraient tous comme leur maitre ${ }^{4}$.

Porter une attention particulière au traitement de la nature dans l'œuvre de Kulbak est donc doublement légitime: premièrement, le

\footnotetext{
2 «[...] fun der gezunter frisher primitiver erdishkayt, vos antplekt far undz a naye farbike yidishkayt oyf der groyer litvish-raysisher landshaft - klasishe geshtaltn fun proste natur- un dorf-yidn mitn royen gevirtsikn reyekh fun feld, vald un taykh ». Cf. Zalman Reyzen, Leksikon fun der yidisher literatur, prese un filologye, vol. 3, col. 604605, Vilnius, 1929. Toutes les traductions du yiddish dans cet article ont été réalisées par moi-même.

3 Si le concept de dorf-yid (juif-de-village) était courant dans la culture des juifs d'Europe de l'Est pour désigner des juifs isolés dans les villages au milieu d'une population largement chrétienne, celui d'un natur-yid (juif-de-la-nature) est une invention. Le fait de désigner les figures de Kulbak comme «classiques » relève donc plutôt en l'occurrence du vœu pieux, à savoir, le rêve des territorialistes qui souhaitaient ancrer le destin juif dans les contrées d'Europe de l'Est.

${ }^{4}$ Une partie importante des comptes rendus critiques et des hommages à Kulbak dont nous disposons proviennent de personnes qui l'ont fréquenté de près dans sa période vilniusienne (1923-1928). Nombre de ces textes ont été publiés après la mort du poète dans Di goldene keyt, le grand journal littéraire yiddish d'après-guerre dirigé par Avrom Sutzkever (voir notamment les $n^{\circ} 5,13,43,77,105$ ). Il s'agit soit d'élèves ou de collègues des différents établissements où il a enseigné la littérature, soit de jeunes écrivains réunis sous l'appellation Yung Vilne à partir de 1929 (et dont Sutzkever faisait partie). Parmi ces hommages, méritent d'être mis en avant ceux de deux membres du groupe : Elkhonen Vogler, « Moyshe Kulbak, der dikhter fun royer erd», [« Moyshe Kulbak, le poète de la terre brute »] Di goldene keyt, vol. 43, 1962, p. 103-122; et le poète Perets Miranski qui souligne l'influence de la vision de la nature par Kulbak sur l'ensemble du groupe dans un hommage enregistré à Montréal le 22 février 1975 : https://archive.org/details/ybc-fbr-438_4437 [consulté le 30 septembre 2018]. L'influence de Kulbak sur « Yung Vilne » est décrite par Justin D. Cammy, «Tsevorfene bleter: The Emergence of Yung Vilne», Polin 14 (2001), p. 170-191 (plus spécifiquement p. 175-176).
} 
monde naturel et les paysages lituaniens jouent un rôle si essentiel dans son œuvre que celle-ci peut être analysée en profondeur sous l'angle du traitement de la nature. Deuxièmement, la persistance de ce thème à travers toutes les métamorphoses littéraires traversées par Kulbak fait de lui une figure essentielle pour comprendre comment la nature a été réinvestie par une certaine modernité yiddish dans une tonalité plus métaphysique que lyrique.

\section{La nature dans la littérature yiddish avant Kulbak}

Pour bien comprendre la place éminente de la nature chez Moyshe Kulbak, il n'est pas inutile de rappeler le rapport complexe du yiddish au monde naturel depuis Mendele Moykher Sforim (1835-1917). Dans son autobiographie, « le grand-père » de la littérature yiddish moderne décrit, de manière assez rousseauiste, l'éveil de l'enfant qu'il était aux charmes de la nature (comparée, de façon révélatrice, à une fiancée). Au rebours toutefois de cette perception rousseauiste, cet éveil est perçu sur le registre de la "faute». Observer, aimer la nature relève du «mauvais penchant», n'est pas «juif»: «Le marché et ses boutiques, les marchands et les faiseurs d'affaires, les tavernes, les auberges, les serviteurs et les commis : - tout cela est, en vérité, juif comme il faut, mais la terre, les champs tout autour sont goys, sont à eux, qu'ils soient bien distingués ! ${ }^{5}$. Le rapport à la nature est donc d'emblée perçu sur le registre de la transgression. Mendele, dont la prose est parsemée de mémorables descriptions de la nature, a créé un précédent essentiel : se tourner vers l'écriture, vers la littérature profane, c'est dans le même mouvement s'opposer au monde juif du shtetl et s'ouvrir à la nature, qui est son opposé.

Chanter la nature ne relève donc pas d'abord de l'évidence pour l'écrivain yiddish, mais presque du combat, d'un mouvement d'ouverture et de découverte lié à une profonde revendication (individuelle et collective). Mendele fut le premier à s'approprier la nature en la judaïsant. Pour que la nature qu'il décrit éveille des sentiments familiers

\footnotetext{
${ }^{5}$ Mendele Moykher Sforim, Shloyme Reb Khayims, dans Ale verk fun Mendele Moykher Sforim, Varsovie, Farlag « Mendele », 1928, vol. 18, p. 62 : « Der mark mit di kleytn, mit di sokhrim un meklers, mit di shenkn, akhsanyes mit di meshorsim un faktoyrim di ale zaynen, emes take, in der guter yidishkayt, ober di erd, di felder arum un arum zaynen dokh goyishe, zeyere, lehavdl ». Voir également l'incipit du roman Fishke der krumer [Fishké, le boîteux] : Mendele y connaît le même combat intérieur que l'enfant Abramovitsh, luttant contre son envie d'admirer les beautés de la nature.
} 
chez ses lecteurs, il la dote non seulement d'attributs humains mais, parfois, proprement juifs : "Sur une branche en hauteur, la pie se balance, et on la croirait, de loin, habillée d'un châle de prière blanc doté de bandes azurées sur le devant, et elle prie en se secouant $»^{6}$. Ces passages lyriques de Mendele, où pointent encore l'humour et la veine picaresque de l'écrivain, vont déboucher à la veille de la Première Guerre mondiale sur un véritable lyrisme fait d'adulation pour une nature dont la judaïsation est fréquente au point d'en devenir stéréotypée.

Les représentants les plus éminents de ce type d'écriture sont le romancier Sholem Asch (1880-1957) et le poète Dovid Eynhorn (18861973) qui sont tous deux désignés par Kulbak lui-même en 1918, dans un rare essai critique, comme les pionniers du lyrisme yiddish ${ }^{7}$. Ce que Kulbak percevait de vindicatif et d'incisif dans l'évocation de la nature chez les «classiques", n'est plus observable chez les poètes de cette « nouvelle " génération (les générations s'enchaînent vite dans ces années de grands bouleversements). Ces écrivains, que l'on peut qualifier de néo-romantiques, dépeignent la vie juive du shtetl, et celle de l'individu juif, comme se déroulant en harmonie avec la nature environnante. En 1904, le court roman A shtetl de Sholem Asch (que l'auteur désignait sous le nom de poème) a, de ce point de vue, été perçu comme une véritable révolution, car jamais le rapport des juifs d'Europe de l'Est à leur environnement (naturel mais aussi social) n'avait été dépeint de façon aussi idyllique. Ainsi de l'épisode où le rebbe hassidique du shtetl bénit le renouveau naturel au printemps dans la forêt : «Tout, à l'entour, était en prière, suppliait et remerciait Dieu, et le

\footnotetext{
${ }^{6}$ Mendele Moykher Sforim, Kleyn-Mendele, éd. Joseph Kutzenogy, p. 174. La citation provient de Fishke der krumer: "Oyf a tsvaygl inderhoykh vigt zikh a soroke, oyszeendik fundervaytns vi ongeton in a vays talesl mit pasn tkheyles front un davnt, shoklendik zikh ».

${ }^{7}$ Moyshe Kulbak, "Dos yidishe vort», initialement publié dans Der veker (Minsk, 1918) et republié dans Moyshe Kulbak, Oysgeklibene Shriftn, éd. Shmuel Rozhanski, Buenos Aires, coll. Musterverk fun der yidisher literatur n ${ }^{\circ} 67$, p. 298-304. De façon révélatrice, Kulbak cite dans cet article une description de la nature par Y. L. Peretz, tirée du recueil Khsidish [Hassidique], mais c'est pour insister sur le style «inquiet, bouillant, convulsif » de l'auteur, dénué de la tendresse du véritable lyrisme. Il trouve ce dernier chez les disciples du grand écrivain, Asch et Eynhorn, ainsi que dans la poésie folklorique. Ce bref essai est d'autant plus intéressant qu'il nous donne une rare occasion d'observer son approche à la fois informée et passionnée de la littérature yiddish, telle qu'il devait l'enseigner à ses nombreux étudiants. Voir le témoignage de l'un d'entre eux, Arthur Lermer, enregistré à Montréal le 11 février 1977: https://archive.org/details/ybc-fbr-601_4600 [consulté le 30 septembre 2018]. Cedernier a été publié par la suite dans: Arthur Lermer, Un dokh - dem morgnroyt antkegn, Tel Aviv, Y. L. Peretz farlag, p. 207-228.
} 
ciel se rapprochait de la terre - la terre du ciel, une assemblée de juifs, une assemblée d'arbres. Les arbres se balançaient avec les hommes $»^{8}$. Quelques années plus tard, en 1909, le premier recueil de Dovid Eynhorn, Shtile Gezangen [Chansons calmes], exprimait la même communion dans le domaine de la poésie lyrique.

Lorsque Kulbak commence à écrire, il est directement sous l'influence de ce type de lyrisme ${ }^{9}$. Mais très vite, les grands bouleversements apportés par la révolution russe de 1917 et l'effervescence créatrice qui en découle, avec une exaltation des tendances modernistes, vont changer radicalement son style. La nature, plus que jamais, est au centre de ses écrits mais ce n'est plus une nature personnifiée, judaïsée, familière et rassurante. En ceci, Kulbak est dans la logique du modernisme poétique yiddish tel que Yankev Glatshteyn l'a évoqué en s'exclamant : «Hélas, avec combien d'amer dégoût, / Nous nous sommes précipités loin des arbres en prière / Loin du triste paysage juif à la Mendele ${ }^{10}$. Et pourtant, Kulbak ne s'est pas tourné comme ses collègues new-yorkais vers la réalité urbaine, ni, comme certains poètes du groupe Khalyastre, vers l'évocation de la violence et de la cruauté du monde contemporain ${ }^{11}$. Il pouvait encore, à la fin des années 1920, être vu par Zalmen Reyzen comme le chantre des «Juifs de la nature $»^{12}$ :

\footnotetext{
${ }^{8}$ Sholem Asch, A shtetl, Vilnius, Kletzkin, 1920, p. 60-61 : «Alts arum hot gedavnt, gebetn un gedankt got, un der himel hot zikh genentert tsu der erd, di erd - tsum himel, an eyde yidn, an eyde beymer. Di beymer hobn zikh mitgeshoklt ».

${ }^{9}$ L'un de ses tout premiers poèmes, «Shterndl», publié en 1916 dans Letste nayes, s'inscrit tout à fait dans cette lignée : des vers légers et mélodiques inspirés de chansons populaires, un thème chargé d'émotion (la lettre du front d'un soldat à sa famille), une personnification de l'étoile qui devient une estafette. Le poème, extrêmement populaire, adapté en chanson, a même été parodié. Voir Z. Reyzen, Leksikon, op. cit., col. 601 et Dovid Katz, "A vilner parodye oyf Moyshe Kulbaks bavust lid 'Shterndl'»: http://defendinghistory.com/keepsakes-of-old-jewish-vilna-44. [consulté le 30/09/2018].

${ }^{10}$ Yankev Glatshteyn, "Ovntike yidn », dans Benjamin et Barbara Harshav, American Yiddish Poetry, A bilingual anthology, Berkeley, Los Angeles, Londres, University of California Press, 1986, p. 352. « Vey, mit vos far a bitere fardrosn / Mir zaynen gelofn fun di davnendike beymer, / Fun troyerikn, yidishn Mendele-landshaft ». Ce poème, qui date de 1949, a la forme d'un examen de conscience. Glatshteyn évoque ici son état d'esprit et celui de ses camarades poètes lors de la fondation du mouvement In zikh en 1919, eux qui refusaient que leur poésie soit limitée à des thèmes et à des images juifs. Après la Shoah, au contraire, la réalité juive s'impose à lui dans toute sa terrible force.

${ }^{11}$ Khalyastre est l'un des nombreux regroupements d'artistes (peintres et écrivains) modernistes qui ont marqué le début des années 1920 parmi les juifs d'Europe de l'Est. Fondé sur l'individualisme et la révolte, il réunissait, entre autres poètes, Melekh Ravitsh, Perets Markish, Uri Tsvi Grinberg. Voir Karolina Szymaniak (éd.), Warszawska awangarda jidysz, Gdańsk, Słowo / obraz terytoria, 2005.

${ }^{12}$ Voir note 3.
} 
c'est cet apparent paradoxe que nous nous proposons maintenant d'étudier.

\section{Le poète de la « terrestrialité » [« erdishkayt »]}

Il est évident que la nature ne disparaît pas de l'œuvre des poètes modernistes après le premier conflit mondial, même si les anciens procédés de judaïsation, de familiarisation, sont en général rejetés. On peut même affirmer que la place remarquable de la nature dans la poésie moderniste est liée à des circonstances sociologiques et biographiques partagées : l'immense majorité des écrivains yiddish, au premier rang desquels Kulbak, ont été éduqués, au moins partiellement, dans les bourgades (voire les villages) de l'arrière-pays, à proximité immédiate de la campagne. La nature de la plaine ukrainienne, des forêts lituaniennes, des steppes russes a donc joué un rôle essentiel dans la formation de leur sensibilité $^{13}$. Chez Kulbak, cependant, la nature devient rapidement bien plus qu'un objet poétique, que l'origine d'un épanchement lyrique.

Dans son premier recueil, Shirim [chants / poèmes], publié en 1920, il exprime déjà un désir de fusion individuelle avec le monde naturel - «Monde / [...] Je veux embrasser ton corps puissamment sauvage / De plantes / Et de pierres [...] ${ }^{14}{ }^{14}$ exprimant jusqu'au désir d'identification avec la roche inanimée, fût-elle même « [...] une pierre de tes pierres dans un cimetière ${ }^{15}$. Il y a dans ces premiers vers beaucoup de l'enthousiasme rimbaldien du jeune homme errant sur les routes, figure chère à Kulbak, et illustrée de façon canonique dans la chanson plus tardive "Ikh bin a bokher a hultay » [Je suis un garçon, un fripon $]^{16}$. Le poète, se représentant lui-même comme enivré de nature,

\footnotetext{
${ }^{13}$ Nous ne pouvons mentionner ici que quelques noms de poètes de la génération de Kulbak chez qui la nature joue un rôle essentiel : Dovid Hofstein, Perets Markish, Rokhl Korn, etc. Chacun d'entre eux a sa forme particulière de lyrisme mais aucun ne partage la vision épique, voire métaphysique, de Kulbak, originalité que l'on retrouvera ensuite chez un poète comme Avrom Sutzkever.

${ }^{14}$ Moyshe Kulbak, Shirim, Vilnius, Farlag fun dem fareyn fun di yidishe literatn un zhurnalistn in Vilne, 1920, p. 27 : «Velt / [...] Ikh vil arumnemen dayn mekhtik-vildn guf / Fun flantsn un geshteynen [...]».

${ }^{15}$ Ibid., p. 28 : « [...] a shteyn fun dayne shteyner / Oyf a beys-oylem lign... ». Ce sont là les vers concluant le poème.

${ }^{16}$ Moyshe Kulbak, Naye lider, Varsovie, Farlag Kulturlige, 1922, p. 7. Le poème ouvre le recueil, ce qui souligne son importance et se trouve intégré au roman Moshiekh ben Efrayim. Composé sur un mètre typique des chansons populaires, il sera effectivement mis en musique. On en trouvera la partition dans: Moyshe Kulbak, Oysgeklibene shriftn, op. cit., p. 31.
} 
recourt à des images frappantes qui lui resteront associées : «Je suis soleil. Je suis rosée. Je me promène, chêne en frac $»^{17}$.

Kulbak est donc un maître de l'évocation des sous-bois, des cours d'eau, des champs, usant d'un vocabulaire riche, n'hésitant pas à recourir à des néologismes, à mimer morphologiquement et syntaxiquement les réalités qu'il dépeint. Souvent, il adopte une perspective grossissante sur les phénomènes naturels, leur conférant un aspect presque fantastique. Il en est ainsi du poème «In a yodlovn vald » [Dans une forêt de sapins], inclus dans son deuxième recueil Naye Lider (1922): «[...] Dans l'amoncellement de branche à branche / Osseuses / Dans l'entrelac du tronc et des racines / Moussues... / Et profond est le fourré chevelu / Glébeux - / C'est un débordement amaniteux / Près des souches / Vertement barbues ${ }^{18}$.

Même dans le long poème «Shtot» [Ville], écrit en 1919 et qui permit à Kulbak de percer en tant que poète moderniste, la nature est loin d'être absente. Cette création en vers de longueur variable, dont le critique Shmuel Niger regrettait le caractère parfois obscur ${ }^{19}$, a marqué les esprits par son ton exalté, par sa façon d'annoncer une ère nouvelle (les allusions à la révolution russe y sont transparentes) qui sera l'œuvre « des jeunes gens de bronze / [...] saisis dès lors d'une envie / d'apaiser / le dépit / des années / perdues à jamais $»^{20}$. Dans ces vers, où s'enchaînent les images fortes de la ville, de son tumulte, de ses bouleversements mais aussi l'évocation voilée de rencontres plus personnelles (avec deux femmes Olye et Miryem, l'une au nom slave,

17 «Kh'bin zun. Kh'bin toy. Kh'gey um a demb in frak», Moyshe Kulbak, Shirim, op. cit., p. 29. L'image est peut-être inspirée du très célèbre recueil de Maïakovsky datant de 1915, Un nuage en pantalon.

${ }^{18}$ Moyshe Kulbak, Naye Lider, op. cit., p. 9 : «In dem tsunoyfgenem fun tsvayg tsu tsvayg / Di knokhike, / In dem geflekht fun shtam un vortsln / Mokhike... / Un tif iz di gedikhtenish di horike, / Di feterdike - / Es iz a kvelenish a mikhomorike / Bay di kortshes / Di grinberdike...» Kulbak utilise ici très librement la souplesse morphologique du yiddish qui permet, par des suffixations et des préfixations, le passage systématique du substantif à l'adjectif, ainsi que la création de mots composés. Certains termes (feterdike, mikhamorike, grinberdike) sont clairement des créations de l'auteur. Tout cela, ajouté à la souplesse du vers, donne le sentiment d'une nature protozoaire, bouillonnante et infiniment créatrice.

${ }_{19}$ Shmuel Niger, Yidishe shrayber in sovet-rusland, New York, Shmuel Niger Bukh komitet, p. 69-75. Les pages 69 à 125 de cet ouvrage réunissent les critiques que Niger, fidèle lecteur de Kulbak, a consacrées à l'écrivain entre 1921 et 1934.

${ }^{20}$ Ibid., p. 73. «Un bronzene yungen / bafaln iz demolt a viln / tsu shtiln / dem tsorn / fun yorn / Vos zaynen farlorn - ». Selon la notice du Leksikon de Reyzen, cette partie $\mathrm{du}$ poème était devenue un véritable hymne révolutionnaire pour la jeunesse socialiste juive : Zalmen Reyzen, Leksikon, op. cit. : col. 602. 
l'autre au nom juif), le poète se définit de façon très personnelle : «C'est une frontière que mon moi / entre forêts et villes $»^{21}$. Et, loin d'être univoque dans sa louange du changement, le poème semble bien s'achever sur une fuite loin des tumultes apportés par le «rouge chevalier, compagnon de la mort » vers les « forêts, vertes éternités $»^{22}$.

C'est qu'une constante parcourt toute l'œuvre de Kulbak: son ancrage dans son territoire d'origine. Or celui-ci n'est pas fortement urbanisé. Les noms de lieux qui apparaissent le plus souvent dans son œuvre, dans les poèmes comme dans les romans, Kobilnik (Kobylnik/Narach), Disne (Dzisna), Krivitsh (Kryvichy), Kreve (Kreva), Luninyets (Luninets), Druye (Druya), sont des petites villes éparpillées sur le territoire de la Biélorussie occidentale (Mayrev Raysn), l'essentiel de l'action se déroulant non pas en leur sein, mais dans la campagne environnante ${ }^{23}$. Cette topographie souligne le caractère fortement localisé de l'imaginaire de Kulbak, et comme ces noms ont des consonnances slaves, ils contribuent à créer un sentiment d'ancrage du monde juif dans le territoire.

Mais Kulbak va plus loin, il entend susciter une forme d'intégration du monde juif dans une nature perçue essentiellement comme non juive (revenant ainsi à la perception classique de la nature comme envers du shtetl, comme univers peuplé de paysans chrétiens). Il s'agit là d'un point essentiel et qui fait toute l'originalité de sa représentation de la nature : la campagne et le monde naturel tels qu'il les représente restent le domaine des goyim, des Slaves, et pourtant tous deux sont parcourus, apprivoisés ou hantés par les héros juifs de ses œuvres. Pour rendre possible ce paradoxe, Kulbak retire à ses personnages juifs ce qui fait, dans la vision traditionnelle du monde ashkénaze, leur judéité ; non pas tant l'aspect rituel du judaïsme (même si, effectivement, il en reste peu de traces), qu'un éthos perçu alors comme distinguant radicalement les juifs des

${ }^{21}$ Ibid., p. 75 : « S’iz a grenets mayn ikh / Tsvishn velder un shtet ».

${ }^{22}$ Ibid., p. 79 : «Velder, velder, grine eybigkaytn» et page 80, le dernier vers du poème : «Der toyt-bagleyter, royter rayter, rayt ! ». A. Leyeles, le poète moderniste de New York et cofondateur du mouvement des introspectivistes (inzikhistn) disait de ce poème qu'il aurait pu être écrit dans la mégalopole américaine et reconnaissait en Kulbak un frère en expérimentation poétique. Voir Moyshe Kulbak, Oysgeklibene shriftn, op. cit., p. 306.

${ }^{23}$ Ces noms sont souvent placés à des endroits stratégiques : le titre (Le Childe Harold de Dzisna), l'incipit (« $\mathrm{O}$, le grand-père de Kobylniki », début du poème Raysn), le nom des personnages des Justes dans les romans modernistes (Berele de Luninets, etc.). Pour trouver la correspondance des noms yiddish de ville aux noms slaves, le linguiste Paul Glasser a créé une ressource fort utile : https://pglasserblog.wordpress.com/ [consulté le 30/09/2018]. 
populations (surtout) paysannes qui les entouraient: une attention tournée vers les choses de l'esprit (la Torah), une maitrise des passions, un mépris de la vie instinctive mue par les besoins élémentaires du corps, un évitement des contacts avec l'étranger et un désintérêt pour le monde naturel. Les juifs de Kulbak sont donc naturels également au sens où leur vie se rapproche des instincts sans que cela n'entraîne une réprobation, bien au contraire.

\section{Le mythe des « Juifs de la nature »}

Dès son premier recueil, dans un poème intitulé «Tsvishn felder " [Parmi les champs], l'écrivain met en scène la rencontre du «je poétique » avec un paysan (situation qui se reproduit dans «Ikh bin a bokher a hultay $»^{24}$ ): s'engage entre eux un dialogue où les premiers mots de chaque réplique sont en polonais pour bien montrer que ce n'est pas en yiddish qu'a lieu la conversation. D'abord engagée sur le thème de l'injustice sociale, celle-ci débouche sur un monologue en forme de confession du poète dans lequel il fait part de ses rapports intimes au monde. Tristesse et exaltation alternent avant de trouver un exutoire dans certains matins d'extase : «Tu tombes sur la terre nue / Tu t'enivres du fumier / de la sève qui coule / Et fermente / Et tu t'exclames à pleins poumons / 'Il existe un tel monde ! / Et moi, l'idiot, je ne le savais pas' $[\ldots] »^{25}$. Que le dialogue s'engage avec un paysan dans une langue slave n'est pas anodin : la nature chez Kulbak n'est peuplée que de goyim à l'exception des héros de ses poèmes et des lamedvovnikes, figures mythologiques des Justes. Le sujet lyrique ou les protagonistes des poèmes narratifs évoluent dans un univers qui n'a rien de juif: ils rencontrent des paysans, des bergers, se prennent de passion pour des femmes non-juives : l'oncle Avrom du poème Raysn [Biélorussie (1922)]

\footnotetext{
${ }^{24}$ Voir Rachel Selig, Strangers in Berlin, Modern Jewish Literature Between East and West, Ann Arbor, University of Michigan Press, 2016, p. 83-86. Rachel Seelig interprète le dialogue final du poème "Ikh bin a bokher a hultay » comme la marque d'un déracinement spécifiquement juif. L'interprétation nous semble erronée, en particulier si l'on compare ce poème à celui plus ancien «Tsvishn felder»: le protagoniste comprend la question en polonais (et non en biélorusse, sauf erreur de notre part), mais y répond par du charabia car il est espiègle et souhaite exprimer son détachement des affaires du monde. Le polonais était très parlé dans la région natale de Kulbak.

${ }^{25}$ Moyshe Kulbak, Shirim, op. cit., p. 35 : «Falstu tsu tsu royer erd : / Verst farshikert funem mist, / Funem zaft vos flist / Un yert, / Un shrayst oys fun gantser brust : / 'S'iz a velt aza faran! / Un ikh, der nar, hob nit gevust' ».
} 
et Bere dans le poème Benye un Bere oyfn shlyakh [Benye et Bere sur la route (1927)] s'éprennent tous deux d'une Nastasye (Nastye dans le deuxième cas) au nom slave évocateur.

Avant de nous intéresser à Raysn, qui est probablement le sommet poétique de la carrière de l'écrivain (sans conteste s'il s'agit d'analyser son rapport à la nature), le deuxième long poème narratif, Benye un Bere, mérite quelques mots. Il appartient à une autre période de la carrière du poète, stylistiquement parlant. Kulbak y adopte le ton héroïcomique qu'il prolongera en Union Soviétique (où il émigre en 1928) pour narrer de façon critique sa période berlinoise dans son long poème intitulé Disner Tshayld-Harold [Childe Harold de Diszna (1933)]. Adoptant ce style ironique, Kulbak reprend également une forme poétique classique: Benye un Bere est composé de sizains de tétramètres iambiques (sauf le dernier vers qui n'a que deux mètres). Pour la narration, il s'agit clairement d'une réécriture du roman épique de Mendele Moykher Sforim Kitser masoes Binyomin hashlishi [Les Voyages de Benjamin III]. Simplement, les héros de Kulbak ne rêvent pas, comme ceux de Mendele, de découvrir le monde mythique des récits traditionnels, ni de retrouver les dix tribus perdues; ils correspondent mieux aux aspirations $\mathrm{du} \mathrm{XX}^{\mathrm{e}}$ siècle : ce sont des bandits révolutionnaires qui rêvent, à grands renforts de beaux discours et de grands coups de poings, d'abolir la propriété. Les œuvres de Kulbak hésitent ainsi entre une temporalité abstraite, liée à un passé mythique (Raysn, Moshiekh Ben Efrayim) et une temporalité davantage ancrée dans le présent puisqu'elle intègre la problématique révolutionnaire, mais qui demeure néanmoins imprécise et comme figée (Benye un Bere, Montog).

Si les pérégrinations de Benye et Bere à travers la campagne biélorusse donnent l'occasion au poète d'offrir de nombreuses descriptions lyriques de la nature, les protagonistes, empêtrés à la fois dans leurs discours socialistes et leurs mésaventures grotesques (ils se font rosser plus d'une fois) ne profitent guère de la beauté environnante. Benye va même jusqu'à désigner ridiculement le monde naturel en employant le jargon à la mode : « Ô humanité sans feu ni lieu, / Ton sort mérite d'être pleuré : / La profession du bec dort dans de doux nids, / Dans de chaudes tanières - les syndicats de la forêt, / Seul l'homme à l'homme ne veut / toujours pas s'habituer ${ }^{26}$. Les deux anti-héros, mus

\footnotetext{
${ }^{26}$ Moyshe Kulbak, Ale verk fun Moyshe Kulbak, Vilnius, Kletskin, 1929, vol. 2, p. 87 : «- O, mentshhayt, on a heym un on a dakh, / Es loynt dayn mazl tsu baveynen, - / In
} 
en permanence par la colère et l'insatisfaction, ne retrouvent un contact fort avec la nature qu'au paroxysme de leur désespoir. C'est le cas de Benye dans la section « Près d'une rivière ». C'est aussi le cas lorsque les deux pieds nickelés rencontrent un vieux berger, qu'ils saluent bien sûr en polonais. Bere supplie alors celui-ci de le laisser souffler dans sa corne : « [...] Ô, grand-père, / Laisse-moi seulement souffler un peu dans ta corne / Je veux ici, pour le mouton et pour la vache, / Entonner un chant de mon enfance $»^{27}$. Le berger ayant accepté, s'ensuit une complainte élégiaque ayant pour refrain « Le champ est Dieu, la corne est l'orant $»^{28}$. La nature continue de jouer un rôle d'interlocuteur privilégié et fait l'objet d'une quasi-sanctification. Ce serait un contresens que d'interpréter cette œuvre de Kulbak comme une satire des idées révolutionnaires (auxquelles l'écrivain était au contraire attaché); il exprime bien plutôt une constante de sa pensée : le malheur de l'homme provient, comme d'une faute originelle, de son détachement du monde naturel, d'où la vanité, et le ridicule, de bon nombre de ses actions ${ }^{29}$.

C'est à cette mythologie personnelle que Kulbak a réussi à donner des proportions épiques dans son poème Raysn [Biélorussie], publié en 1922. Il s'y invente, en effet, une généalogie imaginaire (ou en grande partie fantasmée) et y dépeint son grand-père, ses seize oncles et son père (formant tous ensemble le nombre 18, symbolique de la vie en hébreu) comme des natur-yidn, des Juifs-de-la-nature pour reprendre le terme inventé par Zalmen Reyzen dans son Leksikon ${ }^{30}$. L'enthousiasme

veykhe nestn shloft der shnobl-fakh, / In nares vareme - di vald fareynen, / A mentsh nor tsu a mentshn vil zikh alts / Nit tsugeveynen ».

${ }^{27}$ Ibid., p. 98 : « [...] O, zeydenyu ! / Du loz mir nor a bloz ton in dayn horn, / Ikh vil do far dem sheps un far der ku / A zung ton epes fun di kinder-yorn ».

${ }^{28}$ Ibid., p. 99 : « Dos feld iz got, der horn iz der beter ».

${ }^{29}$ Voir Shmuel Niger, op. cit., p. 91 . Le critique voyait même dans ce texte l'inspiration du grand poème d'Alexandre Blok Les douze : la même ambiguïté s'y retrouve, mais Kulbak demeure d'un lyrisme bien plus classique et la révolution est, dans ce poème qui se conclut aux portes de la ville, aussi réelle que les romans de chevalerie de Don Quichotte.

${ }^{30}$ En effet, Kulbak, selon sa biographie confirmée par le témoignage de ses proches, a grandi dans un milieu attaché à l'étude de la Torah : « Moyshe Kulbak a précocement quitté sa maison et s'en est allé dans l'inconnu gris. Il voulait alors devenir rabbin et s'est rendu dans les yeshives [institution d'étude avancée du Talmud et des textes sacrés] de Sventsyan [Swenciany] et de Volozhin [Valozhyn]». Elkhonen Vogler, « Moyshe Kulbak » art. cit., p. 111. On est très loin de l'univers dépeint par Kulbak dans Raysn puisque toute l'existence et toutes les aspirations de la famille y sont tournées vers la terre. L'expression natur-yidn paraît mieux correspondre à la réalité du texte de Kulbak que celle de dorf-yidn [Juifs de village] également proposée par Reyzen. En effet, la famille au cœur du poème Raysn vit bien dans un village mais on 
exprimé par Reyzen pour ces figures «classiques » n'est peut-être pas seulement dû à la sensibilité territorialiste (voire régionaliste) qui était alors en plein développement ${ }^{31}$ : le mot « classique » peut aussi renvoyer à la réussite stylistique de Kulbak, qui incarne, dans une tonalité noble, la simplicité de ses ancêtres supposés, comme en témoigne l'incipit : «Ô, mon grand-père de Kobylniki est un juif tout simple / Un paysan avec une fourrure, avec une hache, avec un cheval / Et mes seize oncles et mon père - / Des Juifs simples, des Juifs comme des mottes de terre $\rangle^{32}$.

La noblesse $\mathrm{du}$ ton résulte tout à la fois des invocations, des anaphores, des parallélismes, du lexique soutenu mais aussi de l'ampleur $\mathrm{du}$ mètre (dont la longueur varie, essentiellement, du tétramètre à l'hexamètre). Kulbak recourt volontiers au style biblique, comme dans la bénédiction que le grand-père prodigue à ses fils à la veille de sa mort dans la douzième et dernière section : «Rakhmil, qui peut te tenir tête dans la prairie ! / Ta faux dans l'herbe était comme un jaillissement de feu, / Les serpents dans les marais, les oiseaux dans leurs nids te connaissent./ Que la bénédiction repose sur ton étable et sur ta grange ! ${ }^{33}$. C'est là la deuxième de trois bénédictions : le premier fils est lié à la terre (l'agriculture), le second aux champs (l'élevage), le troisième aux rivières (la pêche). Cette réécriture évidente du passage de la Genèse (chapitre 49) où Jacob bénit ses fils, produit une sorte de nouvelle alliance dans laquelle le grand-père lie ses fils à la terre, aux champs, aux rivières de Biélorussie.

Kulbak réussit à mêler emphase et concrétude, souffle épique et corporalité. Il y a, dans ce long poème, comme dans d'autres pièces plus courtes de l'auteur, une tendance à se rattacher au primitivisme dont la

ne la voit que dans sa masure ou dans la nature. La figure classique du dorf-yid est déjà proposée par Mendele Moykher Sforim dans la personne de "Khone der milnitser» [Khone le meunier] et de sa famille, dans son autobiographie, voir Mendele Moykher Sforim, Shloyme reb Khayims, op. cit., chap. 5. N'oublions pas que cette famille apparaît dans le shtetl à l'occasion des « Jours Terribles », autrement dit, elle apparaît sous l'angle de la religiosité juive. Les dorf-yidn font souvent l'objet d'un traitement satirique dans la littérature yiddish, pour leur ignorance religieuse qui n'a d'égale que leur volonté de rester juifs malgré leur isolement.

${ }^{31}$ Voir l'article «Landkentenish » de la Yivo encyclopedia rédigé par David Roskies : http://www.yivoencyclopedia.org/article.aspx/Landkentenish [consulté le 5/10/2018].

${ }^{32}$ Moyshe Kulbak, Ale verk, op. cit., vol. 2, p. 37 : «O, der zeyde fun Kobilnik iz a yid a posheter, / A poyer mit a pelts un mit a hak un mit a ferd / Un mayne zekhtsn feter un mayn tate - / Yidn proste, yidn vi di shtiker erd ».

${ }^{33}$ Ibid., p. 61. « Rakhmil, ver kon zikh mit dir oyf der lonke farmestn ! / Geven iz dayn kose in groz, vi a fleytsl fun fayer, / Dikh kenen di shleng in di zumpn, di feyglen in zeyere nestn. / Di brokhe zol ruen bay dir inem shtal un in shayer!» 
mode traversait de nombreuses avant-gardes européennes. «Plusieurs des mouvements 'primitivistes' du début du siècle se revendiquent d'un retour au passé historico-mythique de leur propre pays $»^{34}$. On identifie une quête semblable chez Kulbak ${ }^{35}$, mais c'est là que le paradoxe devient criant : vers quelle " origine », quelle « primitivité » doit-il se tourner? D'un côté, la nature biélorusse nourrit sa sensibilité la plus profonde. De l'autre, nul plus que cet écrivain yiddish n'est sensible à l'ancrage de sa langue et de sa culture dans une autre spatialité définie par l'héritage biblique et kabbalistique ${ }^{36}$. Dès lors, voulant chanter l'intégration de ses héros à la nature de leur pays dans Raysn, Kulbak fait comme si ces juifs n'étaient pas en diaspora, comme si ces juifs faisaient partie inhérente de ce monde. Lorsque la grand-mère, présentée comme une véritable déesse de la fertilité dans la deuxième section, meurt, c'est tout le village, le pope Vassili compris, qui la pleure en polonais : « La vieille Shlyomikhe n'est plus là ! » ${ }^{37}$.

Une intégration aussi idéale est en fait conçue comme une coincindentia contrariorum, comme une rencontre des contraires qui ne peut avoir lieu que sur le plan mythique. C'est dans le même esprit que Kulbak publiait toute une série de poèmes intitulée " $\mathrm{A}$ dude » [Une douda], du nom d'un instrument à vent traditionnel ressemblant à la cornemuse, et sous-titrée : «La lamentation du vieux Biélorusse $)^{38}$. Cette complainte reprend bien des traits du modernisme dans sa déclinaison primitiviste : le son «dou, dou, dou, dou » se répète de façon régulière scandant, de sa sonorité vide de sens, l'histoire contée par le

\footnotetext{
${ }^{34}$ Voir Isabelle Krzywkowski, "Le Temps et l'Espace sont morts hier ». Les Années 1910-1920. Poésie et poétique de la première avant-garde, Paris, Éditions L'Improviste, 2006, p. 196, (chapitre intitulé : «Le primitivisme dans la poésie des avant-gardes historiques $\gg)$.

${ }^{35}$ Régine Robin, dans son introduction à sa traduction des Zelminyaner où elle évoque le cas de Khlebnikov, écrit que Moyshe Kulbak est «envers et contre tout un néoprimitiviste ». Moïshe Kulbak, Les Zelminiens, Paris, Seuil, 1988, p. 13. Rachel Ertel, quant à elle, dans son introduction à la traduction, par Bernard Vaisbrot, de Montog, parle d'un «primitivisme panthéiste ». Moïshe Kulbak, Lundi, Lausanne, L'Âge d'Homme, 1982, p. XV.

${ }^{36}$ Rien n'est plus typique de ce point de vue que le célèbre poème «Vilne » [Vilnius (1926)]. La ville aux cent clochers devient, sous la plume de Kulbak, entièrement et inconditionnellement juive, l'envers de sa représentation de la nature: " A seyfer iz yede shteyn - a parmet yede vant $[\ldots]$ » [ « Chaque pierre est un livre de prière - chaque mur un parchemin [...] », Moyshe Kulbak, Oysgeklibene shriftn, op. cit., p. 50.

${ }^{37}$ Moyshe Kulbak, Ale verk, op. cit., p. 49 : « Niema, niema, yuzh staroy Shlyomikhe » (septième section). Shlyomikhe signifie ici «la femme de Shloyme». On apprend par la même occasion le nom du grand-père.

${ }^{38}$ Ibid., p. 25.
} 
vieux barde. C'est le récit, esquissé de façon impressionniste, de la fin d'un monde : celui des tribus primitives, autrefois protégées par le vieux dieu slave Bialun, dont la place a été prise par un nouveau Dieu, Jésus, lequel a fait raser aux hommes leurs barbes, source de leur force. Le chanteur dialogue avec les arbres, les oiseaux, la rivière Vilia, et tous lui confirment la triste nouvelle. Dans la septième section, « Jésus », on peut lire : «Le nouveau Dieu nous a demandé : / - Qui est votre père ? / Qui est votre mère, qui - vos frères ? / Ô notre père est la forêt de sapin, / Notre mère - la Vilia dans sa robe de soie / Et nos frères - le corbeau brun et la chouette --$\rangle^{39}$. Quoi de plus naturel pour un poète biélorusse d'imaginer un tel âge d'or préchrétien ? Mais pour un poète yiddish, la chose est nécessairement, ostensiblement, artificielle. Ni l'auteur, ni le lecteur ne peuvent oublier que ce passé fantasmé n'est pas le leur, que la langue qui l'exprime n'est même pas slave.

Dans Raysn, Kulbak effectue un tour de force semblable: il « naturalise », il «biélorussise » ses ancêtres. Ceux-ci ne sont plus définis que par le travail exténuant des champs, que par celui de gardiens de chevaux, ou de conducteurs de radeaux sur la rivière. La nature est leur élément et Kulbak aime la décrire lorsqu'elle est indéfinie, changeante : dans les sous-bois, dans le brouillard, au coucher du soleil. La beauté de la nature secoue de part en part le grand-père du poème : ainsi en est-il dans la quatrième section où Kulbak décrit la façon dont le vieil homme conduit les radeaux de bois sur la rivière avec ses nombreux fils : "Les sucs coulent de la terre si bien qu'une ivresse s'empare de tous les membres, / Une vie s'immisce, muette, à travers les herbes, à travers les racines et les branches, / Si bien que le grand-père n'y résiste plus : il s'y met, oh, il ne peut plus se taire, / Il lance un meuglement : 'Au diable ton père!' et puis encore / 'Au diable ta mère!' [...] ${ }^{40}$. Le Juif-de-la-nature, tel que l'invente Kulbak, est ici présenté dans toute sa splendeur : il est entièrement traversé par la sève qui nourrit toute chose vivante et sa joie fruste, mais intense, s'exprime vulgairement dans un juron qui commence, de façon révélatrice, par un mot slave.

\footnotetext{
${ }^{39}$ Ibid, p. 31 : « Es hot der nayer got bay undz gefregt : / - Ver iz ayer tate ? / Ver iz ayer mame, ver - di brider ayere ? / O, undzer tate iz der vald der yodlover, / Undzer mame di Vilie in zaydenem kleyd, / Un di briderlekh, der broyner rob un di sove - - ».

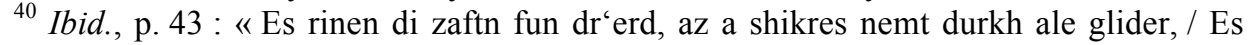
shpart zikh a lebn a shtumer durkh grezer, durkh vortslen un tsvaygn, / Az s'halt mer nit oyset der zeyde, er tut zikh a nem, o, er kon mer nit shvaygn, / Derlangt er a reve : 'Trastsya in dayn tatn!' un nokh a mol vider / 'Trastsya in dayn mamen !' ».
} 
Seuls trois personnages sont vraiment caractérisés dans Raysn : le grand-père, la grand-mère et l'oncle Avrom. Le grand-père est peint en patriarche, en grand ordonnateur. La grand-mère est féconde et juste. L'oncle Avrom est, quant à lui, l'homme de la forêt (il y garde les chevaux), mu par une passion physique pour Nastasye « la plus belle du village ». Le jeu amoureux des deux amants est présenté, dans la section huit, aussi naturellement que celui d'animaux qui se poursuivent en se courtisant. Et quand, dans le froid de l'hiver, le grand-père et les oncles languissent dans leur masure comme la nature sous le gel (section 10), c'est le chant de l'amant rempli de désir qui redonne vie et joie à la compagnie : «Il se mit à secouer sa chevelure vers le haut, vers le bas, / (c'est qu'il avait aperçu en rêve Nastasye, la non-juive) / Il se dressa de tout son long et poussa un grognement, / Comme un étalon qui se languit d'une jument en chaleur ${ }^{41}$. La passion toute physique d'Avrom est une force vitale dont il parvient à partager les bienfaits par la force de son chant $^{42}$.

Mais ce qui fait l'étrange beauté de ce poème, c'est que, tout en ayant complètement «naturalisé » ses personnages, Kulbak rejudaïse son texte en profondeur en le dotant, à plusieurs reprises, d'une résonance biblique. Ainsi, ce même Avrom fait un rêve semblable à celui de Joseph. Mais tandis que le héros de la Genèse voyait le soleil, la lune et onze étoiles se prosterner devant lui, Avrom voit dix-sept étoiles autour de la lune, et l'une d'entre elle, plus brillante, tombe soudain dans le bois moussu. Alors : "Avrom a compris un certain rêve lointain » ${ }^{43}$. Ainsi, tandis que Joseph était destiné à régner sur sa famille, le destin d'Avrom est de plonger dans la mousse de la forêt. C'est le rêve du destin d'Israël qui se trouve entièrement redessiné.

Mais, encore une fois, Kulbak n'est pas dupe du caractère mythique, voire illusoire, de son idylle juive biélorusse. Et comme pour faire entendre une autre voix, légèrement discordante, il insère dans son poème de tonalité noble, deux chansons folkloriques : l'une typiquement

\footnotetext{
${ }^{41}$ Ibid., p. $55:$ : Hot er a treysl geton di tshuprine aroyf un arunter, / (dos hot er derzen inem kholem Nastasye di goye), / Er hot zikh avekgeshtelt oys un gegebn a reytshe / Azoy vi an oger, vos benkt nokh a klyatshe a heyse ».

${ }^{42}$ Pour cette raison, nous ne pouvons que rejeter l'analyse que Marc Caplan offre de la mort de la grand-mère comme étant en partie causée par la relation d'Avrom avec cette non-juive. Voir Marc Caplan : « Belarus in Berlin, Berlin in Belarus : Moyshe Kulbak's 'Raysn' and 'Meshiekh ben-Efrayim' between nostalgia and apocalypse », dans Gennady Estraikh et Mikhail Krutikov (dir.), Yiddish in Weimar Berlin; at the Crossroads of Diaspora Politics and Culture, Leed, Maney Pub., 2010, p. 194.

${ }^{43}$ Moyshe Kulbak, Ale verk, vol. 2, op. cit., p. 47.
} 
juive (section 9), l'autre artificiellement biélorusse (section 11) ${ }^{44}$. Tandis que les passages narratifs privilégient des mètres longs, les chansons ont des mètres beaucoup plus brefs. La première intitulée " Der feter Itse » [L'oncle Itse], représente un personnage de tailleur (y a-t-il métier plus juif?) qui parcourt le pays de village en village pour repriser les vêtements. Le nomadisme de cet oncle, par ailleurs absent du reste du poème, ainsi que son métier artisanal jure avec la peinture des autres personnages. La chanson «biélorusse» s'intitule "Antoshe shpilt oyf der bandure » [Antosha joue sur la bandoura]. Comme " Une douda », elle est rattachée à un instrument folklorique et décrit une période ancienne puisqu'elle met en scène le duc de Kreva (lieu historique de la première union, au Moyen Âge, entre la Lituanie et la Pologne).

L'histoire contée à grands traits est assez comique : le vieux duc a un garçon d'écurie qui séduit ses deux filles et lui procure ainsi deux petits bâtards. Il part alors se cacher dans la forêt. Cette chanson qui pourrait être vue comme un simple divertissement, de couleur locale, avant la scène solennelle de la mort du grand-père, prend une signification plus forte si l'on s'arrête sur le nom du garçon d'écurie : Dmitruk Byadulya. Il ne fait aucun doute que Kulbak a en tête l'auteur juif Shmuel Plavnik, son contemporain. Lié au jeune mouvement nationaliste biélorusse, celui-ci publiait alors, en biélorusse, des poésies et des contes nourris du folklore biélorusse sous le pseudonyme Źmitrok Biadula. Kulbak pensait-il qu'un juif prêtant sa plume au nationalisme biélorusse ne saurait produire que des écrits bâtards ? C'est fort possible et la chanson d'Antosha devait résonner comme une moquerie du célèbre écrivain pour beaucoup de contemporains. Dans tous les cas, en introduisant ces deux chansons, Kulbak rappelle à ses lecteurs que ses « Juifs-de-la-nature » ne sont qu'un mythe, avec tout ce que les mythes comportent d'imperfection et de fantastique ${ }^{45}$.

\footnotetext{
${ }^{44}$ La chanson «juive» sera effectivement mise en musique. Voir Moyshe Kulbak, Oysgeklibene shriftn, op. cit., p. 67.

${ }^{45}$ Malgré ces précautions, l'image construite par Kulbak a tant fasciné ses lecteurs, même les plus avisés, elle répondait à un besoin si fort d'identification au paysage local, que nombreux sont ceux qui ont vu dans Raysn une peinture de juifs, sinon réels, du moins idéaux. Yankev Botoshansky écrivait, dans son introduction à une réédition d'après-guerre des deux romans expressionnistes de Kulbak: "Personne n'a aussi profondément judaïsé la nature que ne l'a fait Kulbak. Mendele Moykher Sforim a judaïsé la nature extérieurement, Kulbak l'a fait dans son intériorité la plus profonde ». Voir Moyshe Kulbak, Moshiekh ben Efrayim un Montik, Buenos Aires, D. Lerman, 1950, p. 11. Notre analyse montre la difficulté de soutenir une telle affirmation sans la nuancer et l'argumenter. Mais bien souvent, la critique yiddish a voulu prendre, à la
} 


\section{Conclusion}

Nous avons centré notre analyse sur l'œuvre poétique de Kulbak car c'est elle qui présente, dans toute sa pureté, sa vision mythologisée de la nature. Mais il serait également passionnant d'analyser la façon dont le rapport essentiel des Justes et des mendiants à la nature est au centre des expérimentations audacieuses de ses romans modernistes des années 1920 (Moshiekh Ben Efrayim et Montog), et de voir en quoi la présentation presque animalisée des protagonistes est à la source de l'humour subversif du grand roman soviétique Di Zelminyaner ${ }^{46}$. En définitive, la capacité qu'a eue Kulbak de se réinventer tout au long de sa courte carrière, dans des genres variés, est liée à la constance d'une vision du monde ancrée au plus profond de l'écrivain : l'existence d'un lieu intime, viscéral, à la nature de sa région natale, la Biélorussie, dans sa pureté élémentaire. Que ce lien ne soit pas évident à mettre en valeur, à mythifier, pour un écrivain juif de langue yiddish, n'a fait qu'encourager Kulbak à trouver des solutions esthétiques nouvelles et extrêmement originales. La constance de cette inspiration naturelle, élémentaire, est remarquable et l'on peut convoquer comme témoins des écrits fort variés, tels le conte pour enfant « Der vint vos iz geven in kas » [Le vent qui était en colère] dont le protagoniste est le vent lui-même, le cycle de poèmes expressionnistes «Velfishe lider » [Chants de loup] où le Je poétique s'identifie à la solitude famélique du loup, ou la nouvelle de la fin des années 1920 "Munye der foygl-hendler un Malkele zayn vayb» [Munye, le marchand d'oiseaux, et Malkele sa femme] dans laquelle les oiseaux sont bien plus qu'un élément du décor ${ }^{47}$.

L'influence de Kulbak et de sa relation à la nature a été majeure sur les poètes de Yung Vilne. Son disciple, Elkhonen Vogler, reprend grandement à son compte, tout en en déplaçant le sens, son admiration pour les paysages de Lituanie et de Biélorussie ${ }^{48}$. Mais il nous semble

lettre, le mythe créé par Kulbak comme un symbole de l'harmonie régnant entre juifs, biélorusses et le territoire qui les unissait.

${ }^{46}$ Nous prévoyons de publier un article sur ce sujet dans un prochain numéro de Tsafon.

${ }^{47}$ Moyshe Kulbak, Dos vint vos iz geven in kas, Vilnius, Tsentrale shul-organizatsye, 1921 ; "Velfishe lider» dans Moyshe Kulbak, Ale verk, op. cit, vol. 2, p. 9-13; " Munye der foygl-hendler un Malkele zayn vayb», dans Chone Shmeruk (éd.), $A$ shpigl oyf a shteyn, antologye [Un miroir sur une pierre, anthologie], Jerusalem, Magnes, 1987, p. 556-566. Cette anthologie mérite d'être recommandée pour la qualité de l'édition des textes et de l'appareil critique.

${ }^{48}$ Voir Mikhail Krutikov, "Rajsn, Belarus v modernistskoj poezii na idishe » dans

« Glubokoe : pamjat' o evrejskom mestechke », Moscou, Sefer, 2017, p. 141-150. 
également qu'un poète de la stature d'Avrom Sutzkever doit beaucoup à Kulbak, surtout au début de sa carrière, dans son recueil Sibir [Sibérie]. On retrouve chez lui une capacité à exprimer son émerveillement devant ce que la nature a de plus primitif et de plus élémentaire ainsi qu'une tendance à accorder aux phénomènes naturels un sens plus profond, presque métaphysique. La Sibérie de Sutzkever est un mythe de l'enfance et de la découverte du monde, comme la Biélorussie de Kulbak est le mythe du juif naturel, complètement enraciné dans son territoire. 